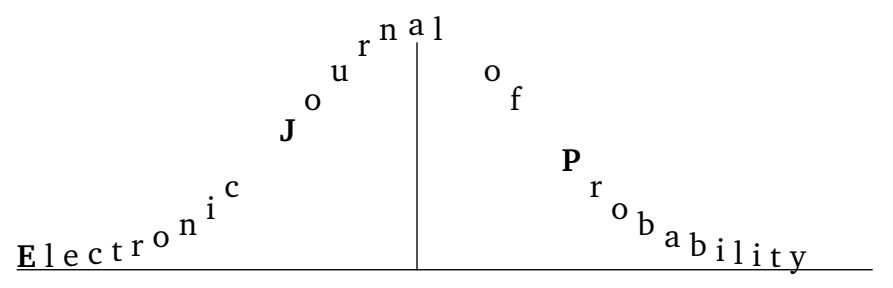

Vol. 14 (2009), Paper no. 77, pages 2264-2266.

Journal URL

http://www.math.washington.edu/ ejpecp/

\title{
The time constant vanishes only on the percolation cone in directed first passage percolation
}

\author{
Yu Zhang* \\ University of Colorado
}

\begin{abstract}
We consider the directed first passage percolation model on $\mathbf{Z}^{2}$. In this model, we assign independently to each edge $e$ a passage time $t(e)$ with a common distribution $F$. We denote by $\vec{T}(0,(r, \theta))$ the passage time from the origin to $(r, \theta)$ by a northeast path for $(r, \theta) \in$ $\mathbf{R}^{+} \times[0, \pi / 2]$. It is known that $\vec{T}(\mathbf{0},(r, \theta)) / r$ converges to a time constant $\vec{\mu}_{F}(\theta)$. Let $\vec{p}_{c}$ denote the critical probability for oriented percolation. In this paper, we show that the time constant has a phase transition at $\vec{p}_{c}$, as follows:

(1) If $F(0)<\vec{p}_{c}$, then $\vec{\mu}_{F}(\theta)>0$ for all $0 \leq \theta \leq \pi / 2$.

(2) If $F(0)=\vec{p}_{c}$, then $\vec{\mu}_{F}(\theta)>0$ if and only if $\theta \neq \pi / 4$.

(3) If $F(0)=p>\vec{p}_{c}$, then there exists a percolation cone between $\theta_{p}^{-}$and $\theta_{p}^{+}$for $0 \leq \theta_{p}^{-}<\theta_{p}^{+} \leq$ $\pi / 2$ such that $\vec{\mu}(\theta)>0$ if and only if $\theta \notin\left[\theta_{p}^{-}, \theta_{p}^{+}\right]$. Furthermore, all the moments of $\vec{T}(\mathbf{0},(r, \theta))$ converge whenever $\theta \in\left[\theta_{p}^{-}, \theta_{p}^{+}\right]$.

As applications, we describe the shape of the directed growth model on the distribution of $F$. We give a phase transition for the shape at $\vec{p}_{c}$.
\end{abstract}

Key words: directed first passage percolation, growth model, and phase transition.

AMS 2000 Subject Classification: Primary 60K 35.

Submitted to EJP on September 22, 2008, final version accepted September 30, 2009.

*Research supported by NSF grant DMS-4540247. 


\section{Introduction of the model and results.}

In this directed first passage percolation model, we consider the vertices of the $\mathbf{Z}^{2}$ lattice and the edges of the vertices with the Euclidean distance 1 . We denote by $\mathbf{L}^{2}$ these edges. We assign independently to each edge a non-negative passage time $t(e)$ with a common distribution $F$. More formally, we consider the following probability space. As the sample space, we take $\Omega=\prod_{e \in \mathrm{L}^{2}}[0, \infty)$, whose points are called configurations. Let $\mathbf{P}=\prod_{e \in \mathrm{L}^{2}} \mu_{e}$ be the corresponding product measure on $\Omega$, where $\mu_{e}$ is the measure on $[0, \infty)$ such that

$$
\mu_{e}(t(e) \leq x)=F(x) .
$$

The expectation with respect to $\mathbf{P}$ is denoted by $\mathbf{E}(\cdot)$. For any two vertices $u$ and $v$ in $\mathbf{Z}^{2}$, a path $\gamma$ from $u$ to $v$ is an alternating sequence $\left(v_{0}, e_{1}, v_{1}, \ldots, v_{i}, e_{i+1}, v_{i+1}, \ldots, v_{n-1}, e_{n}, v_{n}\right)$ of vertices $v_{i}$ and edges $e_{i}$ between $v_{i}$ and $v_{i+1}$ in $\mathbf{L}^{2}$, with $v_{0}=u$ and $v_{n}=v$. For a vertex $u$, its northeast edges from $u$ are denoted by $u=\left(u_{1}, u_{2}\right)$ to $\left(u_{1}+1, u_{2}\right)$ or to $\left(u_{1}, u_{2}+1\right)$. Given a path $\left(v_{0}, e_{1}, v_{1}, \ldots, v_{i}, e_{i+1}, v_{i+1}, \ldots, v_{n-1}, e_{n}, v_{n}\right)$, if each edge $e_{i}$ is a northeast edge from $v_{i}$, the path is called northeast, or directed. For short, we denote northeast edges or northeast paths by NE edges or NE paths.

Given a path $\gamma$, we define its passage time as

$$
T(\gamma)=\sum_{e \in \gamma} t(e)
$$

For any two vertices $u$ and $v$, we define the passage time from $u$ to $v$ by

$$
T(u, v)=\inf \{T(\gamma)\}
$$

where the infimum is over all possible paths $\{\gamma\}$ from $u$ to $v$. We also define

$$
\vec{T}(u, v)=\inf \{T(\gamma)\}
$$

where the infimum is over all possible NE paths $\{\gamma\}$ from $u$ to $v$. If there does not exist a NE path from $u$ and $v$, we simply define

$$
\vec{T}(u, v)=\infty .
$$

A NE path $\gamma$ from $u$ to $v$ with $T(\gamma)=\vec{T}(u, v)$ is called an optimal path of $\vec{T}(u, v)$. We need to point out that the optimal path may not be unique. If we focus on a special configuration $\omega$, we may write $\vec{T}(u, v)(\omega)$, instead of $\vec{T}(u, v)$.

In addition to vertices on $\mathbf{Z}^{2}$, we may also consider points on $\mathbf{R}^{2}$. In particular, we often use the polar coordinates $\{(r, \theta)\}=\mathbf{R}^{+} \times[0, \pi / 2]$, where $r$ and $\theta$ represent the radius and the angle between the radius and the $X$-axis, respectively. We may extend the definition of passage time over $\mathbf{R}^{+} \times[0, \pi / 2]$. For $(r, \theta)$ in $\mathbf{R}^{+} \times[0, \pi / 2]$, we define $\vec{T}(\mathbf{0},(r, \theta))=\vec{T}(\mathbf{0},(\lfloor r \sin \theta\rfloor,\lfloor r \cos \theta\rfloor))$. Similarly, $\vec{T}(u, v)$ can be defined for any $u, v \in \mathbf{R}^{2}$. Moreover, with this extension, for any points $u$ and $v$ in $\mathbf{R}^{2}$, we may consider a path of $\mathbf{Z}^{2}$ from $u$ to $v$.

Given an angle $\theta \in[0,2 \pi]$, by a subadditive argument, if $\mathbf{E} t(e)<\infty$, then

$$
\lim _{r \rightarrow \infty} \frac{1}{r} T(\mathbf{0},(r, \theta))=\lim _{r \rightarrow \infty} \frac{1}{r} \mathbf{E} T(\mathbf{0},(r, \theta))=\inf _{r} \frac{1}{r} \mathbf{E} T(\mathbf{0},(r, \theta))=\mu_{F}(\theta) \text { a.s. and in } L_{1} \text {. }
$$


We call $\mu_{F}(\theta)$ a time constant. Furthermore, by the same subadditive argument, for an angle $\theta \in$ $[0, \pi / 2]$,

$$
\lim _{r \rightarrow \infty} \frac{1}{r} \vec{T}(\mathbf{0},(r, \theta))=\lim _{r \rightarrow \infty} \frac{1}{r} \mathbf{E} \vec{T}(\mathbf{0},(r, \theta))=\inf _{r} \frac{1}{r} \mathbf{E} \vec{T}(\mathbf{0},(r, \theta))=\vec{\mu}_{F}(\theta) \text { a.s. and in } L_{1} .
$$

We also call $\vec{\mu}_{F}(\theta)$ a time constant. By the subadditive argument again, we know (see Proposition 2.1 (iv) in Martin (2004)) that

$$
\vec{\mu}_{F}(\theta) \text { is finite and convex in } \theta .
$$

In general, we require that $t(e)$ has a finite first moment or $m$-th moment. However, we sometimes require the following stronger tail assumption:

$$
\operatorname{Eexp}(\eta \tau(e))<\infty \text { for } \eta>0 \text {. }
$$

Recall the undirected first passage percolation model for $\{T(u, v)\}$. Kesten (1986) showed that there is a phase transition at critical probability, $p_{c}$, of bond percolation for a time constant. More precisely, he showed that time constant $\mu_{F}(\theta)$ vanishes if and only if $F(0) \geq p_{c}$. Therefore, $F(0)>p_{c}, F(0)=p_{c}$, and $F(0)<p_{c}$ are called the supercritical, the critical, and the subcritical phases, respectively. It is natural to examine a similar situation for the directed first passage percolation model. In this paper, our focus is that there is also a phase transition for $\vec{\mu}_{F}(\theta)$ at critical probability, $\vec{p}_{c}$, of directed bond percolation. We will demonstrate for the supercritical and critical phases, which are quite different from the undirected first passage percolation model (see Kesten and Zhang (1997), and Zhang (1995)). We will also examine the subcritical phase, which is similar to the undirected model (see Kesten (1986)).

1.1. Supercritical phase. We now focus on the supercritical phase: $F(0)>\vec{p}_{c}$. Before introducing our results, we would like to introduce a few basic oriented percolation results. If we rotate our lattice counterclockwise by $45^{\circ}$ and extend each edge by a factor of $\sqrt{2}$, the new graph is denoted by $\mathscr{L}$ with oriented edges from $(m, n)$ to $(m+1, n+1)$ and to $(m-1, n+1)$. Each edge is independently open or closed with probability $p$ or $1-p$. An oriented path from $u$ to $v$ is defined as a sequence $v_{0}=u, v_{1}, \cdots, v_{m}=v$ of points of $\mathscr{L}$. The path has the vertices $v_{i}=\left(x_{i}, y_{i}\right)$ and $v_{i+1}=\left(x_{i+1}, y_{i+1}\right)$ for $0 \leq i \leq m-1$ such that $y_{i+1}=y_{i}+1$ and $v_{i}$ and $v_{i+1}$ are connected by an oriented edge. An oriented path is open if each of its edges is open. For two vertices $u$ and $v$ in $\mathscr{L}$, we say $u \rightarrow v$ if there is an oriented open path from $u$ to $v$. For $A \subset(-\infty, \infty)$, we denote the following random subset by

$$
\xi_{n}^{A}=\left\{x: \exists x^{\prime} \in A \text { such that }\left(x^{\prime}, 0\right) \rightarrow(x, n)\right\} \text { for } n>0 .
$$

The right edge for this set is defined by

$$
r_{n}=\sup \xi_{n}^{(-\infty, 0]}(\sup \emptyset=-\infty) .
$$

By a subadditive argument (see Section 3 (7) in Durrett (1984)), there exists a non-random constant $\alpha_{p}$ such that

$$
\lim _{n \rightarrow \infty} \frac{r_{n}}{n}=\lim _{n} \frac{\mathrm{E} r_{n}}{n}=\alpha_{p} \text { a.s. and in } L_{1}
$$


where $\alpha_{p}>0$ if $p>\vec{p}_{c}$, and $\alpha_{p}=0$ if $p=\vec{p}_{c}$, and $\alpha_{p}=-\infty$ if $p<\vec{p}_{c}$. Now we rotate the lattice back to $\mathbf{Z}^{2}$. If $p \geq \vec{p}_{c}$, the percolation cone is the cone between two polar equations $\theta=\theta_{p}^{\mp}$ in the first quadrant, where (see Marchand (2002))

$$
\theta_{p}^{\mp}=\arctan \left(\frac{1 / 2 \mp \alpha_{p} / \sqrt{2}}{1 / 2 \pm \alpha_{p} / \sqrt{2}}\right) .
$$

Note that if $p=\vec{p}_{c}$, then the percolation cone shrinks to the positive diagonal line. In fact, for any point $(r, \theta)$ with $\theta \in\left[\theta_{p}^{-}, \theta_{p}^{+}\right]$, it can be shown (see Lemma 3.3 in Yukich and Zhang (2006)) that

$$
\mathbf{P}[\exists \text { a NE zero-path from the origin to }(r, \theta)]>C \text {. }
$$

In this paper, $C$ and $C_{i}$ are always positive constants that may depend on $F$, but not on $t, r, k$, or $n$. Their values are not significant and may change from appearance to appearance. With these definitions, we have the following theorem regarding the passage time on the percolation cone:

Theorem 1. If $F(0)=p>\vec{p}_{c}$ and $\mathbf{E}(t(e))^{m}<\infty$ for $m \geq 1$, then there exists $C=C(F, m)$ such that for every $r \geq 0$ and $\theta \in\left[\theta_{p}^{-}, \theta_{p}^{+}\right]$,

$$
\mathbf{E} \vec{T}(\mathbf{0},(r, \theta))^{m} \leq C
$$

In contrast to the passage time on the percolation cone, we have another theorem:

Theorem 2. If $F(0)=p>\vec{p}_{c}$ and $\theta \notin\left[\theta_{p}^{-}, \theta_{p}^{+}\right]$, then there exist positive constants $\delta=\delta(F, \theta)$ and $C_{i}=C_{i}(F, \theta, \delta)$ for $i=1,2$ such that for every $r \geq 0$,

$$
\mathbf{P}[\vec{T}(\mathbf{0},(r, \theta)) \leq \delta r] \leq C_{1} \exp \left(-C_{2} r\right) .
$$

Together with Theorems 1 and 2, we have the following corollary:

Corollary 3. If $F(0)=p>\vec{p}_{c}$ and $\mathbf{E}(t(e))<\infty$, then for $0 \leq \theta \leq \pi / 2$,

$$
\vec{\mu}_{F}(\theta)=0 \text { iff } \theta \in\left[\theta_{p}^{-}, \theta_{p}^{+}\right]
$$

Remark 1. We would like to discuss $\vec{\mu}_{F}(\theta)$ as a function of $F$. Recall that in the general first passage percolation model, Yukich and Zhang (2006) showed that the time constant is not third differentiable in the direction of $\theta_{p}^{ \pm}$. We find out that the same proof together with

$$
T(u, v) \leq \vec{T}(u, v)
$$

can be carried out to show the same result for directed first passage percolation. Here we state the following result but omit the proof. We denote by $\vec{\mu}_{F}(\theta, p)$ the time constant for $F(0)=p$. If $t(e)$ only takes two values 0 or 1 and $F(0)>\vec{p}_{c}$, then

$$
\vec{\mu}_{F}\left(\theta_{p}^{ \pm}, p\right) \text { is not third differentiable in } p .
$$

Except in these two directions, we believe that there is no other singularity. 
Remark 2. Note that $\vec{\mu}_{F}(\theta)$ can also be considered as a function of $\theta$. By the convexity in (1.2), we can show that $\vec{\mu}_{F}(\theta)$ is continuous in $\theta$. We believe that $\theta_{p}^{\mp}$ are also the singularities for $\vec{\mu}_{F}(\theta)$ in $\theta$.

Conjecture 1. If $F(0)>\vec{p}_{c}$, show that $\vec{\mu}_{F}(\theta)$ has singularities at $\theta_{p}^{ \pm}$.

1.2. Critical phase. We focus on the critical phase: $F(0)=\vec{p}_{c}$. Now, as we mentioned, the percolation cone shrinks to the positive diagonal line. Similar to the supercritical phase, we can show the following theorem:

Theorem 4. If $F(0)=\vec{p}_{c}$ and $\theta \neq \pi / 4$, then there exist positive constants $\delta=\delta(F, \theta)$ and $C_{i}=$ $C_{i}(F, \theta, \delta)$ for $i=1,2$ such that for every $r \geq 0$,

$$
\mathbf{P}[\vec{T}(\mathbf{0},(r, \theta)) \leq \delta r] \leq C_{1} \exp \left(-C_{2} r\right) .
$$

The time constant at $\theta=\pi / 4$ has double behaviors: supercritical and subcritical behaviors. First, we show that it has a supercritical behavior:

Theorem 5. If $\mathrm{E} t(e)<\infty$ and $F(0)=\vec{p}_{c}$, then

$$
\vec{\mu}_{F}(\pi / 4)=0
$$

Remark 3. Cox and Kesten used a circuit method (1981) to show the following result, which is a stronger result than (1.7). If $F_{n} \Rightarrow F$, then

$$
\lim _{n \rightarrow \infty} \mu_{F_{n}}(\theta)=\mu_{F}(\theta) .
$$

However, their method cannot be applied for the directed model, since a path may not be directed after using a piece of circuit. By the uniform convergence estimate in (1.9), it is possible to show Cox and Kesten's argument for the directed model.

Together with Theorems 4 and 5, we have the following corollary:

Corollary 6. If $F(0)=\vec{p}_{c}$ and $\mathrm{E}(t(e))<\infty$, then for $0 \leq \theta \leq \pi / 2$,

$$
\vec{\mu}_{F}(\theta)=0 \text { iff } \theta=\pi / 4 \text {. }
$$

To pursue the convergence rate, we need to use the isoperimetric inequality by Talagrand (1995). Denote by $M$ a median of $\vec{T}(0,(r, \theta))$. By Theorem 8.3.1 (see Talagrand (1995)), if (1.3) holds, then for all $r>0$ and $1 \leq x \leq \sqrt{r}$, there exist positive constants $C_{i}=C_{i}(F, \theta)$ for $i=1,2$ such that

$$
\mathbf{P}[|\vec{T}(\mathbf{0},(r, \theta))-\mathbf{E} \vec{T}(\mathbf{0},(r, \theta))| \geq x \sqrt{r}] \leq C_{1} \exp \left(-C_{2} x^{2}\right) .
$$

With this concentration inequality, we can use Alexander's result (1996) to show the following. For all $r$, if (1.3) holds, there exists $C=C(F, \theta)$ such that for all $0<r$

$$
r \vec{\mu}(\theta) \leq \mathbf{E} \vec{T}(\mathbf{0},(r, \theta)) \leq r \vec{\mu}_{F}(\theta)+C \sqrt{r} \log r .
$$


With (1.9) and Theorem 5, if (1.3) holds and $F(0)=\vec{p}_{c}$, then there exists $C=C(F)$ such that

$$
\mathbf{E} \vec{T}(\mathbf{0},(r, \pi / 4)) \leq C \sqrt{r} \log r .
$$

Remark 4. The upper bound might not be tight at the right side of (1.10). In fact, we believe the following conjecture in a much tight upper bound:

Conjecture 2. If (1.3) holds and $F(0)=\vec{p}_{c}$, show that

$$
\mathbf{E} \vec{T}(\mathbf{0},(r, \pi / 4)) \leq C \log r
$$

Note that (1.11) holds for the undirected first passage time (see Chayes, Chayes, and Durrett (1986)). In contrast, the lower bound is more complicated. It might depend on how $F(x) \downarrow F(0)=\vec{p}_{c}$ as $x \downarrow 0$ as the same way as the undirected model (see Zhang (1999)). We believe the following conjecture.

Conjecture 3. There exists $F$ with $F(0)=\vec{p}_{c}$ such that for every $r>0$,

$$
E \vec{T}(0,(r, \pi / 4)) \leq C .
$$

However, we believe that $\vec{T}(0,(r, \pi / 4))$ has a subcritical behavior for other distributions similar to the behavior of undirected passage time. More precisely, we expect

$$
\lim _{r \rightarrow \infty} \mathbf{E} \vec{T}(\mathbf{0},(r, \pi / 4))=\infty
$$

for some $F$ with $F(0)=\vec{p}_{c}$. In fact, we may simply ask the same questions when $t(e)$ only takes 0 and 1 with $F(0)=\vec{p}_{c}$.

Conjecture 4. If $t(e)$ only takes 0 and 1 with $F(0)=\vec{p}_{c}$, show that

$$
C_{1} \log r \leq \mathrm{E} \vec{T}(\mathbf{0},(r, \pi / 4)) \leq C_{2} \log r .
$$

Note that (1.14) is indeed true (see Chayes, Chayes, and Durrett (1986)) for the undirected critical model. Furthermore, Kesten and Zhang (1997) showed a central limit theorem for the passage time in the undirected critical model. Here, we partially verify (1.14) for the directed critical model:

Theorem 7. If $t(e)$ only takes two values 0 and 1 with $F(0)=\vec{p}_{c}$, then

$$
\lim _{r \rightarrow \infty} \mathbf{E} \vec{T}(\mathbf{0},(r, \pi / 4))=\infty .
$$

Remark 5. As we mentioned above, we know the continuity of $\vec{\mu}_{F}(\theta)$ in $\theta$. We believe that there is a power law when $\theta \rightarrow \pi / 4$. More precisely, we assume that $F(0)=\vec{p}_{c}$ and $t(e)$ only takes values 0 and 1.

Conjecture 5. $\vec{\mu}_{F}(\theta) \approx|\theta-\pi / 4|^{\alpha}$ for some $0<\alpha<1$. 
1.3. Subcritical phase. Finally, we focus on the subcritical phase: $F(0)=p<\vec{p}_{c}$. On this phase, we show the following theorem:

Theorem 8. If $F(0)<\vec{p}_{c}$, then there exist positive constants $\delta=\delta(F)$ and $C_{i}=C_{i}(F, \delta)$ for $i=1,2$ such that for every $r>0$ and every $\theta \in[0, \pi / 2]$,

$$
\mathbf{P}[\vec{T}(\mathbf{0},(r, \theta)) \leq \delta r] \leq C_{1} \exp \left(-C_{2} r\right) .
$$

By Theorem 8, there exists $C=C(F)$ such that for all $r$ and $\theta$

$$
\mathbf{E}[\vec{T}(\mathbf{0},(r, \theta))] \geq C r .
$$

With (1.15) and (1.1), we have the following corollary:

Corollary 9. If $\mathrm{E} t(e)<\infty$ and $F(0)<\vec{p}_{c}$, then for every $\theta \in[0, \pi / 2]$,

$$
\vec{\mu}_{F}(\theta)>0
$$

Remark 6. We would like to focus on a special case in the subcritical phase. In fact, Hammersley and Welsh (1965) considered $t(e)+a$ for some real number $a$. They used $F \oplus a(x)=F(x-a)$ to denote the distribution. Clearly, if $a>0$, each edge takes at least a time $a$, so $F \oplus a(0)=0$. Therefore, it is in the subcritical phase. Durrett and Liggett (1981) consider the case where

$$
F \oplus a(a)>\vec{p}_{c}
$$

for undirected passage time $T(u, v)$. When $(r, \theta)$ is in the percolation cone, by (1.5) it can be shown that an optimal path for $T(\mathbf{0},(r, \theta))$ is directed with a positive probability. Thus, by Corollary 3

$$
\lim _{r \rightarrow \infty} \frac{T(\mathbf{0},(r, \theta))}{r}=\mu_{F \oplus a}(\theta)=a
$$

This well known result (see Durrett and Liggett (1981)) is called the flat edge of the shape.

1.4. Shape of the growth model. We may discuss the shape theorem for this directed first passage percolation. Define the shape as

$$
\mathbf{C}_{t}=\left\{(r, \theta) \in \mathbf{R}^{+} \times[0, \pi / 2]: \vec{T}(\mathbf{0},(r, \theta)) \leq t\right\} .
$$

For each $(r, \theta) \in \mathbf{R}^{+} \times[0, \pi / 2]$, by the subadditive argument,

$$
\lim _{s \rightarrow \infty} \frac{1}{s} \vec{T}(\mathbf{0},(s r, \theta))=\lim _{s \rightarrow \infty} \frac{1}{s} \mathbf{E} \vec{T}(\mathbf{0},(s r, \theta))=\vec{\mu}_{F}(r, \theta) \text { a.s. and in } L_{1} .
$$

By (1.1) and (1.17), we know that

$$
r \mu_{F}(\theta)=\mu_{F}(r, \theta) .
$$

With (1.17), we define the directed growth shape as

$$
\mathbf{C}=\left\{(r, \theta) \in \mathbf{R}^{+} \times[0, \pi / 2]: \vec{\mu}_{F}(r, \theta) \leq 1\right\} .
$$


With these definitions, Martin (2004) proved that if $\mathbf{E} t^{2}(e)<\infty$ and

$$
\inf _{r \neq 0} \frac{\vec{\mu}_{F}(r, \theta)}{r}>0
$$

then $\mathbf{C}$ is a convex compact set, and for any $\epsilon>0$,

$$
(1-\epsilon) \mathbf{C} \subset \frac{\mathbf{C}_{t}}{t} \subset(1+\epsilon) \mathbf{C} \text {, eventually with probability } 1 .
$$

The result in (1.19) is called shape theorem. In the subcritical case, for all $0 \leq \theta \leq \pi / 2$, by Corollary $8, \mathrm{C}$ is a convex compact set such that the shape theorem holds. We denote the shape between two angles by

$\mathbf{C}\left(\theta_{1}, \theta_{2}\right)=\left\{(r, \theta) \in \mathbf{R}^{+} \times\left[\theta_{1}, \theta_{2}\right]: \vec{\mu}_{F}(r, \theta) \leq 1\right\}, \mathbf{C}_{t}\left(\theta_{1}, \theta_{2}\right)=\left\{(r, \theta) \in \mathbf{R}^{+} \times\left[\theta_{1}, \theta_{2}\right]: \vec{T}(\mathbf{0},(r, \theta)) \leq t\right\}$

for $0 \leq \theta_{1} \leq \theta_{2} \leq \pi / 2$. Furthermore, we denote by $\rho(\theta)$ the boundary point $(\rho(\theta), \theta)$ of $\mathbf{C}$.

In the supercritical case, by Corollary 3 and (1.19), for any small $\delta>0$,

$$
\mathbf{C}\left(0, \theta_{p}^{-}-\delta\right) \text { and } \mathbf{C}\left(\theta_{p}^{+}+\delta, \pi / 2\right) \text { are convex compact sets }
$$

such that the shape theorem holds:

$$
(1-\epsilon) \mathbf{C}\left(0, \theta_{p}^{-}-\epsilon\right) \subset \frac{\mathbf{C}_{t}\left(0, \theta_{p}^{-}-\delta\right)}{t} \subset(1+\epsilon) \mathbf{C}\left(0, \theta_{p}^{-}-\delta\right)
$$

and

$$
(1-\epsilon) \mathbf{C}\left(\theta_{p}^{+}+\delta, \pi / 2\right) \subset \frac{\mathbf{C}_{t}\left(\theta_{p}^{+}+\delta, \pi / 2\right)}{t} \subset(1+\epsilon) \mathbf{C}\left(\theta_{p}^{+}+\delta, \pi / 2\right)
$$

eventually with probability 1 . On the other hand, by Corollary 3 again,

$$
\mathbf{C}\left(\theta_{p}^{-}, \theta_{p}^{+}\right) \text {and } \lim _{t} \frac{\mathbf{C}_{t}\left(\theta_{p}^{-}, \theta_{p}^{+}\right)}{t} \text { equal the percolation cone. }
$$

In the critical case, for any small $0<\delta$, by Corollary 6 and (1.19),

$$
\mathbf{C}(0, \pi / 4-\delta) \text { and } \mathbf{C}(\pi / 4+\delta, \pi / 2) \text { are convex compact sets }
$$

such that the shape theorem holds:

$$
(1-\epsilon) \mathbf{C}(0, \pi / 4-\epsilon) \subset \frac{\mathbf{C}_{t}(0, \pi / 4-\delta)}{t} \subset(1+\epsilon) \mathbf{C}(0, \pi / 4-\delta)
$$

and

$$
(1-\epsilon) \mathbf{C}(\pi / 4+\delta, \pi / 2) \subset \frac{\mathbf{C}_{t}(\pi / 4+\delta, \pi / 2)}{t} \subset(1+\epsilon) \mathbf{C}(\pi / 4+\delta, \pi / 2)
$$

eventually with probability 1 . On the other hand, by Corollary 6 again,

$$
\mathbf{C}(\pi / 4, \pi / 4) \text { and } \lim _{t} \frac{\mathbf{C}_{t}(\pi / 4, \pi / 4)}{t} \text { equal the positive diagonal line. }
$$




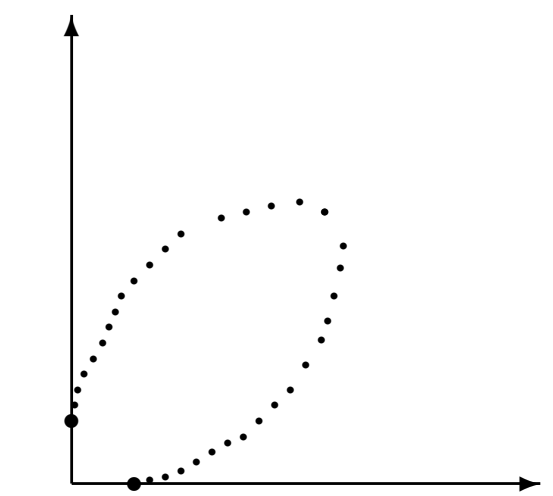

Subcritical phase: $F(0)<\vec{p}_{c}$

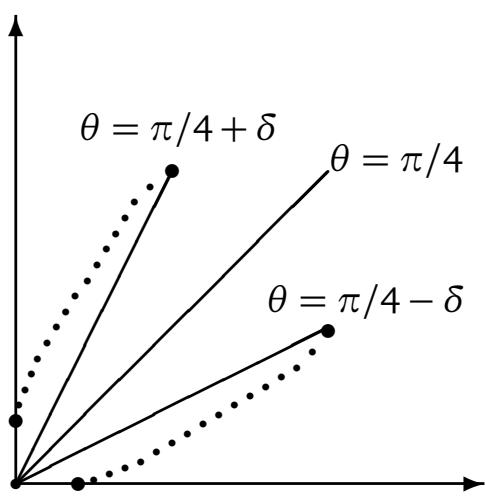

Critical phase: $F(0)=\vec{p}_{c}$

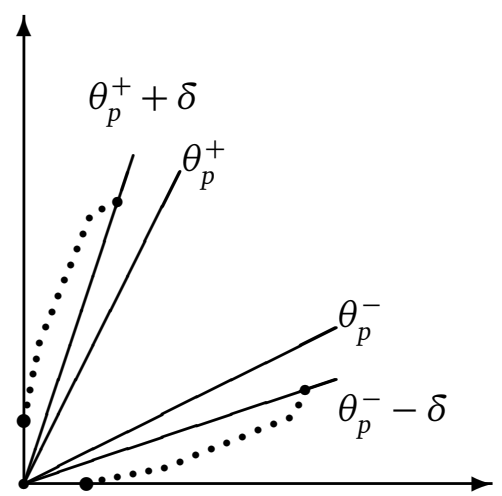

Supercritical phase: $F(0)>\vec{p}_{c}$

Figure 1: The graph shows shape $\mathbf{C}$ in subcritical, critical, and supercritical phases. In the supercritical phase, the right figure, the shape is the percolation cone between two angles $\theta_{p}^{ \pm}$, and the other two parts of the shape are finite. In the critical phase, the middle figure, the percolation cone shrinks to the positive diagonal line. The other two parts of the shape are finite. In the subcritical phase, the left figure, the shape is finite.

In particular, in both the supercritical and critical phases, by Theorems 1 and 2, and Theorem 5 , the continuity of $\mu_{F}(\theta)$ in $\theta$,

$$
\rho(\theta) \rightarrow \infty \text { as } \theta \rightarrow \theta_{p}^{ \pm}
$$

In the subcritical case, by Corollary 9, the shape theorem in (1.19) holds. We can describe the phases of the shapes as Fig. 1.

Remark 7. Since the shape is convex, by (1.25), on the critical and super critical phases, the slope of the line passing through $\left(\rho\left(\theta_{1}\right), \theta_{1}\right)$ and $\left(\rho\left(\theta_{2}\right), \theta_{2}\right)$ cannot be more than $\tan \left(\theta_{p}^{-}\right)$when $\theta_{1}<\theta_{2}<\theta_{p}^{-}$. By symmetry, we have the same property when $\pi / 2 \geq \theta_{1}>\theta_{2}>\theta_{p}^{+}$.

We may relate the directed first passage percolation to the following directed growth model. At time 1 , a cell $\mathbf{A}_{1}$ consists of the unit square with the center at the origin. Each square has four edges: the north, the east, the south, and the west edges. Two squares are connected if they have a common edge. Suppose that at time $n$ we have connected $n$ unit squares, denoted by $\mathbf{A}_{n}$. Let $\partial \mathbf{A}_{n}$ be the boundary of $\mathbf{A}_{n}$. A square is a boundary square of $\mathbf{A}_{n}$ if one of its edges belongs to $\partial \mathbf{A}_{n}$. We collect all the north and the east edges in $\partial \mathbf{A}_{n}$ from the boundary squares. We denote these edges by the northeast edges of $\mathbf{A}_{n}$. At time $n+1$, a new square is added to $\mathbf{A}_{n}$ such that it connected to the northeast edges of $\mathbf{A}_{n}$. The location of the new square is chosen with a probability proportional to the northeast edges of $\mathbf{A}_{n}$.

Now we consider $F$ has an exponential distribution with rate 1. By the same discussion, we can define the directed growth shape $\mathbf{C}_{t}$ and show the shape theorem of (1.19). By a similar computation (see page 131 in Kesten (1986)), we can show that shapes $\mathbf{A}_{n}$ and $\mathbf{C}_{t_{n}}$ have the same distribution 
with

$$
t_{n}=\inf \left\{t: \mathbf{C}_{t} \text { contains } n \text { vertices }\right\} \text {. }
$$

Thus, the shape theorem for $\mathbf{C}_{t}$ implies that $n^{-1 / 2} \mathbf{A}_{n}$ has also an asymptotic shape.

Unlike the undirected model, the oriented percolation model in higher dimensions has been more limited. For example, we cannot define the right edge $r_{n}$ for the oriented percolation model when $d>2$. However, if one could develop a similar argument of the percolation cone as we defined in Section 1.1, our techniques for first passage percolation would apply for higher dimensions.

\section{Preliminaries.}

2.1. Renormalization method. We introduce the method of renormalization in Kesten and Zhang (1990). We define, for a large integer $M$ and $w=\left(w_{1}, w_{2}\right) \in \mathbf{Z}^{2}$, the squares by

$$
B_{M}(w)=\left[M w_{1}, M w_{1}+M\right) \times\left[M w_{2}, M w_{2}+M\right) .
$$

We denote these $M$-squares by $\left\{B_{M}(w): w \in \mathrm{Z}^{2}\right\}$. For a path $\gamma$ (not necessary a directed path) starting from the origin, we denote a fattened $\gamma_{M}$ by

$$
\gamma_{M}=\left\{B_{M}(w): B_{M}(w) \cap \gamma \neq \emptyset\right\}
$$

We denote by $|A|$ the number of vertices inside $A$. Since $\gamma_{M}$ consists of $M$-squares, let $\left|\gamma_{M}\right|$ be the number of $M$-squares in $\gamma_{M}$. By our definition,

$$
|\gamma| \geq\left|\gamma_{M}\right| \text { and }\left|\gamma_{M}\right| \geq \frac{|\gamma|}{M^{2}}
$$

For each $M$-square $B_{M}(w)$, there are eight $M$-square neighbors. We say they are adjacent to $B_{M}(w)$. We denote $B_{M}(w)$ and its eight $M$-square neighbors by $\bar{B}_{M}(w) . \bar{B}_{M}(w)$ is called a $3 M$-square.

If $B_{M}(w) \cap \gamma \neq \emptyset$ and $\bar{B}_{M}(w)$ does not contain the origin of the path $\gamma$, note that $\gamma$ has to cross $\bar{B}_{M}(w) \backslash B_{M}(w)$ to reach $B_{M}(w)$, so $\bar{B}_{M}(w)$ contains at least $M$ vertices of $\gamma$. We collect all $3 M$-squares $\left\{\bar{B}_{M}(w)\right\}$ such that their center $M$-squares contain at least a vertex of $\gamma$. We call these $3 M$-squares center $3 M$-squares of $\gamma$. With these definitions, the following lemma (see Zhang, page 22 (2008)) can be calculated directly.

Lemma 1. For a connected path $\gamma$, if $\left|\gamma_{M}\right|=k \geq 2$, then there are at least $k / 15$ disjoint center $3 M$-squares of $\gamma$.

2.2. Results for oriented percolation. We assign either open or closed to each edge with probability $p$ or $1-p$ independently from the other edges. For two sets $A$ and $B$, if there exists a NE open path from $u \in A$ to $v \in B$, we write $A \rightarrow B$.

First, we focus on the subcritical phase: $p<\vec{p}_{c}$. Let

$$
\mathscr{C}_{0}=\{u: 0 \rightarrow u\} .
$$

Durrett (Section 7, (6) (1984)) showed the following lemma: 
Lemma 2. If $p<\vec{p}_{c}$, then there exist positive constants $C_{i}$ for $i=1,2$ such that

$$
\mathbf{P}\left[\left|\mathscr{C}_{0}\right| \geq n\right] \leq C_{1} \exp \left(-C_{2} n\right) .
$$

Now we focus on the critical and supercritical phases: $p \geq \vec{p}_{c}$. Given two points $u=\left(u_{1}, u_{2}\right)$ and $v=\left(v_{1}, v_{2}\right)$, we define the slope between them by

$$
s l(u, v)=\frac{v_{2}-u_{2}}{v_{1}-u_{1}} .
$$

With these definitions, Zhang (Lemma 3 (2008)) showed the following lemma:

Lemma 3. Let $p \geq \vec{p}_{c}$ and $a \in\left(0, \tan \left(\theta_{p}^{-}\right)\right)$. There exist positive constants $C_{i}=C_{i}(p, a)$ for $i=1,2$ such that for every $u \in \mathbf{R} \times[0, \pi / 2] \cap \mathbf{Z}^{2}$ with $\operatorname{sl}(\mathbf{0}, u) \leq a$,

$$
\mathbf{P}[\mathbf{0} \rightarrow u] \leq C_{1} \exp \left(-C_{2} u_{1}\right)
$$

2.3. Analysis for the shape $C_{t}$. Now we would like to introduce a few geometric properties for $\mathbf{C}_{t}$. In the remainder of Section 2.3, we only consider $t(e)$ when it takes value 0 or 1 with $F(0)=p$. If $t(e)=0, e$ is said to be open or closed otherwise.

Given a set $\Gamma \subset \mathbf{R}^{2}$ that contains at least a vertex of $\mathbf{Z}^{2}$, we let $\Gamma^{\prime}$ be all vertices on $\mathbf{Z}^{2}$ contained in $\Gamma$. It is easy to see that

$$
\Gamma^{\prime} \subset \Gamma \subset\left\{v+(-1,1)^{2}: v \in \Gamma^{\prime}\right\} .
$$

A set $A$ in $\mathbf{Z}^{2}$ is said to be directly connected if there exists a vertex (root) $v \in A$ such that any vertex of $u$ of $A$ is connected from $v$ by a NE path in $A$.

Given a finite directly connected set $\Gamma$ of $\mathbf{Z}^{2}$, we define its vertex boundary as follows. A site $v \in \Gamma$, is said to be a boundary vertex of $\Gamma$ if there exists $u \notin \Gamma$ such that $u$ is adjacent to $v$ by either a north or an east edge. We denote by $\partial \Gamma$ the set of all boundary vertices of $\Gamma$. We also let $\partial_{0} \Gamma$ be all vertices not in $\Gamma$, but adjacent to $\partial \Gamma$ by north or east edges. $\partial_{e} \Gamma$ denotes the set of all NE edges between $\partial \Gamma$ and $\partial_{0} \Gamma$.

We need to work on the following event

$$
\left\{\mathbf{C}_{t}^{\prime}=\Gamma\right\}=\left\{\omega: \mathbf{C}_{t}^{\prime}(\omega)=\Gamma\right\}
$$

With these definitions, Zhang (see propositions 1-3 in Zhang (2006)) proved the following lemmas for undirected first passage percolation. The proofs can be carried out by changing paths to directed paths, so we omit the proofs. In fact, these lemmas are easily understood by drawing a few figures.

Lemma 4. $\mathrm{C}_{t}^{\prime}$ is directly connected.

Lemma 5. For all $v \in \partial \mathbf{C}_{t}^{\prime}, \vec{T}(\mathbf{0}, v)=t$, and for all $u \in \partial_{o} \mathbf{C}_{t}^{\prime}, \vec{T}(\mathbf{0}, u)=t+1$.

Lemma 6. The event of $\left\{\mathbf{C}_{t}^{\prime}=\Gamma\right\}$ only depends on the zeros and ones of the edges in $\Gamma \cup \partial_{o} \Gamma$. 
2.4. Monotone property for the time constant. Finally, we would like to introduce a monotone lemma for the time constant. Comparing two distributions $F_{1}$ and $F_{2}$, we have the following lemma:

Lemma 7. If $\mathrm{E} t(e)<\infty$ and $F_{1}(x) \leq F_{2}(x)$ for all $x$, then for $\theta \in[0, \pi / 2]$,

$$
\vec{\mu}_{F_{2}}(\theta) \leq \vec{\mu}_{F_{1}}(\theta) \text {. }
$$

Proof. Smythe and Wierman (1978) proved the same result in their Theorem 7.12 for undirected first passage percolation. The same coupling argument can be carried out to show Lemma 7 .

\section{Subcritical phase.}

In Section 3, we assume that $F(0)<\vec{p}_{c}$. Since $F$ is right-continuous, we take a small $\epsilon>0$ such that

$$
F(\epsilon)<\vec{p}_{c} .
$$

We say that an edge is open if $t(e) \leq \epsilon$, otherwise $e$ is said to be closed. With (3.1), we know that

$$
\mathbf{P}[e \text { is open }]<\vec{p}_{c} .
$$

Now we work on an optimal path $\gamma$ for $\vec{T}(0,(r, \theta))$. As before, we use $\gamma_{M}$ to denote the squares of $\gamma$. If a square in $\gamma_{M}$ contains a closed edge of $\gamma$, we call the square a bad square. Otherwise, it is a good square. Now we want to count the possible choices of these squares. Note that $\gamma$ is connected, and so is $\gamma_{M}$. We assume that

$$
\left|\gamma_{M}\right|=k \geq 2
$$

Since $\gamma$ is connected, $\gamma_{M}$ is a directed square path such that each square in $\gamma_{M}$ is either directly to the right of or directly above the square. Thus, there are at most $2^{k}$ choices for all possible choices of $\gamma_{M}$ and

$$
k=\left\lfloor\frac{r \sin \theta}{M}\right\rfloor+\left\lfloor\frac{r \cos \theta}{M}\right\rfloor+1 .
$$

By Lemma 1 in Section 2, we know there are at least $\left|\gamma_{M}\right| / 15$ disjoint center $3 M$-squares of $\gamma$. Thus, if there are less than $\left|\gamma_{M}\right| / 30$ bad squares, there are at least

$$
\left|\gamma_{M}\right| / 15-\left|\gamma_{M}\right| / 30 \geq\left|\gamma_{M}\right| / 30
$$

disjoint $3 M$-squares such that their center squares contain an edge of $\gamma$ and all the $M$-squares in these disjoint $3 M$-squares are good. We also call these $3 M$-squares good. When $\gamma_{M}$ is fixed, we select these good $3 M$-squares. There are at most $2^{k}$ choices for these good $3 M$-squares.

For each good $3 M$-square $\bar{B}_{M}(w)$, there exists a NE open path crossing the $3 M$-square from a vertex at the boundary of $B_{M}(w)$ to another vertex at the boundary of $\bar{B}_{M}(w)$. There are at most $4 M$ choices for the starting vertex, and the path contains at least $M$ edges. For a fixed $\bar{B}_{M}(w)$, we denote by $\mathscr{E}_{w}$ the event that there exists a NE open path from $B_{M}(w)$ to the boundary of $\bar{B}_{M}(w)$. By Lemma 2 , there are positive constants $C_{i}=C_{i}(F)$ for $i=1,2$ such that

$$
\mathbf{P}\left[\mathscr{E}_{w}\right] \leq C_{1} M \exp \left(-C_{2} M\right)
$$


Note that $\mathscr{E}_{w}$ and $\mathscr{E}_{u}$ are independent with the same distribution for fixed $w$ and $u$ if $B_{w}(M)$ and $B_{u}(M)$ are two center squares of two disjoint $3 M$-squares. With these observations and (3.3), if we take $M$ large, there exist positive constants $C_{i}=C_{i}(F)$ for $i=1,2$ in $(3.5)$ and $C_{j}=C_{j}(F, M)$ for $j=3,4$ such that

$$
\begin{aligned}
& \mathbf{P}\left[\exists \text { an optimal path } \gamma \text { for } \vec{T}(\mathbf{0},(r, \theta)) \text { with less than }\left|\gamma_{M}\right| / 30 \text { bad squares }\right] \\
\leq & 2^{k} 2^{k} \mathbf{P}\left[\mathscr{E}_{0}\right]^{k / 30} \\
\leq & 4^{k}\left(C_{1} M \exp \left(-C_{2} M\right)\right)^{k / 30} \\
\leq & C_{3} \exp \left(-C_{4} r\right) .
\end{aligned}
$$

Proof of Theorem 8. For the $M$ in (3.5), we select $\delta>0$ such that

$$
\delta<\epsilon\left(60 M^{2}\right)^{-1}
$$

Thus, by (3.6)

$$
\begin{aligned}
& \mathbf{P}[\vec{T}(\mathbf{0},(r, \theta)) \leq \delta r] \\
\leq \quad & \mathbf{P}[\vec{T}(\mathbf{0},(r, \theta)) \leq \delta r, \exists \text { an optimal path } \gamma \text { for } \vec{T}(\mathbf{0},(r, \theta)) \\
& \text { with more than } \left.\left|\gamma_{M}\right| / 30 \text { bad squares }\right]+C_{3} \exp \left(-C_{4} r\right) .
\end{aligned}
$$

Note that if there are more than $\left|\gamma_{M}\right| / 30$ bad squares, then $\gamma$ contains at least $\left|\gamma_{M}\right| / 30$ edges with passage time larger than $\epsilon$. Note also that $\gamma$ is a path from the origin to $(r, \theta)$, so $\gamma$ contains at least $r-1$ edges. Thus, by (2.1) $\gamma$ contains at least $(r-1) /\left(30 M^{2}\right)$ edges with passage time larger than $\epsilon$. Since $\gamma$ is an optimal path, $\vec{T}(0,(r, \theta))$ cannot be less than or equal $\delta r$. Therefore, there exist positive constants $C_{i}(F, \delta)$ for $i=5,6$ such that

$$
\mathbf{P}[\vec{T}(\mathbf{0},(r, \theta)) \leq \delta r] \leq C_{5} \exp \left(-C_{6} r\right) .
$$

Thus, Theorem 8 follows from (3.7).

\section{Outside the percolation cone.}

The proofs for theorems outside the percolation cone also need the method of renormalization. We assume in Theorems 2 and 4 that

$$
F(0) \geq \vec{p}_{c} .
$$

Thus, we say an edge is open if $t(e)=0$ and closed otherwise. With (4.1), we have

$$
\mathbf{P}[e \text { is open }] \geq \vec{p}_{c} .
$$

In this Section, we also denote an optimal path from the origin to $(r, \theta)$ by $\gamma$ and denote the $M$ squares of $\gamma$ by $\gamma_{M}$ for a large $M$. If a square in $\gamma_{M}$ contains an edge $e$ of $\gamma$ with $t(e)>0$, we say the square is a bad square. Otherwise, it is a good square.

Now we count the number of the choices for these squares. Note that $\gamma$ is connected, and so is $\gamma_{M}$. We assume that

$$
\left|\gamma_{M}\right|=\left\lfloor\frac{r \sin \theta}{M}\right\rfloor+\left\lfloor\frac{r \cos \theta}{M}\right\rfloor+1=k \geq 2 \text { and } \exists l \text { bad squares among these } k \text { squares. }
$$


As noticed in Section 3, there are at most $2^{k}$ choices for all possible $\gamma_{M}$. When $\gamma_{M}$ is fixed, we select these bad squares. There are at most

$$
\sum_{l=1}\left(\begin{array}{l}
k \\
l
\end{array}\right) \leq 2^{k}
$$

choices for these bad squares. We list all the bad squares as

$$
S_{1}, S_{2}, \cdots, S_{l}
$$

for $l \leq k$. For each $S_{i}$, the path $\gamma$ will meet the boundary of $S_{i}$ at $v_{i}^{\prime}$ and then use less than $2 M$ edges to meet $v_{i}^{\prime \prime}$, another boundary point of $S_{i}$. We denote the path along $\gamma$ from the origin to $v_{1}^{\prime}$ by $\gamma_{0}$, from $v_{1}^{\prime \prime}$ to $v_{2}^{\prime}$ by $\gamma_{1}, \cdots$, from $v_{l-1}^{\prime \prime}$ to $v_{l}^{\prime}$ by $\gamma_{l-1}$. Note that bad edges are only contained in bad squares, so $\gamma_{i}$ does not contain any bad edge. For a fixed $\gamma_{M}$, and fixed $S_{i}$ and $S_{i+1}$ of $\gamma_{M}$, and fixed $v_{i}^{\prime \prime} \in \partial S_{i}$ and fixed $v_{i+1}^{\prime} \in \partial S_{i+1}$, let $\gamma_{i}\left(v_{i}^{\prime \prime}, v_{i+1}^{\prime}\right)$ be a NE open path from $v_{i}^{\prime \prime}$ to $v_{i+1}^{\prime}$ without using edges in $S_{i}$ and $S_{i+1}$ for $i=0,1, \cdots, l$, where $v_{0}^{\prime \prime}=(0,0)$ and $v_{l+1}^{\prime}=(r, \theta)$. We simply denote by $\left\{\exists \gamma_{i}\left(v_{i}^{\prime \prime}, v_{i+1}^{\prime}\right)\right\}$ the event such that $\gamma_{i}\left(v_{i}^{\prime \prime}, v_{i+1}^{\prime}\right)$ exists. Now we reconstruct a NE fixed open path from $v_{i}^{\prime}$ to $v_{i}^{\prime \prime}$. Let $\mathscr{E}_{i}\left(v_{i}^{\prime}, v_{i}^{\prime \prime}\right)$ be the event that there is a NE open path from $v_{i}^{\prime}$ to $v_{i}^{\prime \prime}$. For $v_{i}^{\prime}, v_{i}^{\prime \prime}$ and $v_{i+1}^{\prime}$ in $\partial S_{i} \cup \partial S_{i+1}$, there might not be NE paths from $v_{i}^{\prime}$ to $v_{i}^{\prime \prime}$ or from $v_{i}^{\prime \prime}$ to $v_{i+1}^{\prime}$. However, in this paper, we only focus on $v_{i}^{\prime}$, $v_{i}^{\prime \prime}$ and $v_{i+1}^{\prime}$ in $\partial S_{i} \cup \partial S_{i+1}$ for $i=0, \cdots, l$ such that the NE paths exist.

Since there exists a NE path with less than $2 M$ edges from $v_{i}^{\prime}$ to $v_{i}^{\prime \prime}$,

$$
\mathbf{P}\left[\mathscr{E}_{i}\left(v_{i}^{\prime}, v_{i}^{\prime \prime}\right)\right] \geq\left(\vec{p}_{c}\right)^{2 M} .
$$

Also, there are at most $(4 M)^{2}$ choices for $v_{i}^{\prime}$ and $v_{i}^{\prime \prime}$ when $S_{i}$ is fixed. After fixing $l$, and $S_{i}$, and $v_{i}^{\prime}$ and $v_{i}^{\prime \prime}$ in $\partial S_{i}$ for $i=1,2, \cdots, l$,

$$
\left\{\bigcap_{i=0}^{l}\left\{\exists \gamma_{i}\left(v_{i}^{\prime \prime}, v_{i+1}^{\prime}\right)\right\}\right\} \text { and }\left\{\bigcap_{i=1}^{l} \mathscr{E}_{i}\left(v_{i}^{\prime}, v_{i}^{\prime \prime}\right)\right\} \text { are independent, }
$$

since two events use the edges in different paths, respectively. Moreover, if

$$
\bigcap_{i=0}^{l}\left\{\exists \gamma_{i}\left(v_{i}^{\prime \prime}, v_{i+1}^{\prime}\right)\right\} \bigcap_{i=1}^{l} \mathscr{E}_{i}\left(v_{i}^{\prime}, v_{i}^{\prime \prime}\right)
$$

occurs, then there exists a NE open path from the origin to $(r, \theta)$. By (4.3), note that $\gamma$ is directed, so

$$
2 r \geq|\gamma| \geq(k-1) M
$$

With these observations, for a small constant $C \in(0,1)$ and each $k$ defined in (4.3), we try to estimate the following probability by fixing $\gamma_{M}$, and then $l$, and then $S_{i}$, and finally $v_{i}^{\prime}$ and $v_{i}^{\prime \prime}$ for 


$$
i=1,2, \cdots, l \text {. }
$$

$\mathbf{P}\left[\exists\right.$ an optimal $\gamma$ for $\vec{T}(\mathbf{0},(r, \theta))$ with $\left|\gamma_{M}\right|=k$ and less than $C k$ bad squares $]$

$$
\begin{aligned}
& \leq 2^{k} 2^{k} \sum_{l=0}^{C k} \sum_{v_{1}^{\prime}, v_{1}^{\prime \prime}, \cdots, v_{l}^{\prime}, v_{l}^{\prime \prime}} \mathbf{P}\left[\bigcap_{i=0}^{l}\left\{\exists \gamma_{i}\left(v_{i}^{\prime \prime}, v_{i+1}^{\prime}\right)\right\}\right] \\
& \leq 4^{k} \sum_{l=1}^{C k} \sum_{v_{1}^{\prime}, v_{1}^{\prime \prime}, \cdots, v_{l}^{\prime}, v_{l}^{\prime \prime}} \mathbf{P}\left[\bigcap_{i=0}^{l}\left\{\exists \gamma_{i}\left(v_{i}^{\prime \prime}, v_{i+1}^{\prime}\right)\right\}\right]\left(\vec{p}_{c}\right)^{-2 C k M} \prod_{i=1}^{l} \mathbf{P}\left[\mathscr{E}_{i}\left(v_{i}^{\prime}, v_{i}^{\prime \prime}\right)\right] \\
& \leq 4^{k}\left(\vec{p}_{c}\right)^{-2 C k M} \sum_{i=1}^{C k} \sum_{v_{1}^{\prime}, v_{1}^{\prime \prime}, \cdots, v_{l}^{\prime}, v_{l}^{\prime \prime}} \mathbf{P}\left[\bigcap_{i=0}^{l}\left\{\exists \gamma_{i}\left(v_{i}^{\prime \prime}, v_{i+1}^{\prime}\right)\right\} \bigcap_{i=1}^{l} \mathscr{E}_{i}\left(v_{i}^{\prime}, v_{i}^{\prime \prime}\right)\right] \\
& \leq C k(4 M)^{2 C k} 4^{k}\left(\vec{p}_{c}\right)^{-2 C k M} \mathbf{P}[\exists \text { an open path from the origin to }(r, \theta)],
\end{aligned}
$$

where the sum $\sum_{v_{1}^{\prime}, v_{1}^{\prime \prime}, \cdots, v_{1}^{\prime}, v_{1}^{\prime \prime}}$ is over all possible vertices of $v_{i}^{\prime}, v_{i}^{\prime \prime}$ on the boundary of fixed $S_{i}$ for $i=1, \cdots, l$. Let $u=\left(u_{1}, u_{2}\right)$ be the ending vertex of $\gamma$. If $\theta<\theta_{p}^{-}$, then

$$
\operatorname{sl}(\mathbf{0}, u)=\tan (\theta)<\tan \left(\theta_{p}^{-}\right) .
$$

In addition,

$$
u_{1}=O(r)
$$

Thus by Lemma 3, (4.9), (4.10), and (4.7), there exist positive constants $C_{i}=C_{i}(F, \theta)$ for $i=1,2,3$ such that

$$
\mathbf{P}[\exists \text { an open NE path from the origin to }(r, \theta)] \leq C_{1} \exp \left(-C_{2} r\right) \leq C_{1} \exp \left(-C_{3} M k\right)
$$

If we substitute (4.11) into (4.8), then

$$
\begin{aligned}
& \mathbf{P}\left[\exists \text { an optimal } \gamma \text { for } \vec{T}(\mathbf{0},(r, \theta)) \text { with }\left|\gamma_{M}\right|=k \text { and less than } C k \text { bad squares }\right] \\
\leq & C k(4 M)^{2 C k} 4^{k}\left(\vec{p}_{c}\right)^{-2 C k M} C_{1} \exp \left(-C_{3} M k\right) .
\end{aligned}
$$

Therefore, if we take $C=C\left(\vec{p}_{c}, F, \theta\right)$ small and $M$ large, there exist positive constants $C_{i}=$ $C_{i}(F, \theta, C, M)$ for $i=4,5$ such that for every large $r$ and $k$ defined in (4.3)

$\mathbf{P}\left[\exists\right.$ an optimal $\gamma$ for $\vec{T}(\mathbf{0},(r, \theta))$ with $\left|\gamma_{M}\right|=k$ and less than $C k$ bad squares $] \leq C_{4} \exp \left(-C_{5} r\right)$.

If there exist less than $C|\gamma|$ closed edges for an optimal path $\gamma$, then by (2.1) there are less than $C\left|\gamma_{M}\right|$ bad squares. Therefore, there exist positive constants $C=C(F, \theta)$ and $C_{i}=C_{i}(F, \theta, C)$ for $i=6,7$ such that for all $r \geq 0$,

$$
\begin{aligned}
& \mathbf{P}[\exists \text { an optimal } \gamma \text { for } \vec{T}(\mathbf{0},(r, \theta)) \text { with less than } C|\gamma| \text { closed edges }] \\
\leq & \mathbf{P}\left[\exists \text { an optimal } \gamma \text { for } \vec{T}(\mathbf{0},(r, \theta)) \text { with }\left|\gamma_{M}\right|=k \text { and less than } C k \text { bad squares }\right] \\
\leq & C_{6} \exp \left(-C_{7} r\right)
\end{aligned}
$$

With (4.12), we show Theorems 2 and 4: 
Proofs of Theorems 2 and 4. Suppose that there exists a NE path $\gamma$ from the origin to $(r, \theta)$ with

$$
\vec{T}(\gamma) \leq C_{8} r
$$

for some constant $C_{8}$. Note that if there are more than $C|\gamma|$ closed edges, then there are more than $\mathrm{Cr}$ closed edges. By (4.12), we may use the $C$ such that

$$
\begin{aligned}
& \mathbf{P}\left[\vec{T}(\gamma) \leq C_{8} r\right] \\
=\quad & \mathbf{P}\left[\vec{T}(\gamma) \leq C_{8} r, \gamma \text { with more than } C|\gamma| \text { closed edges }\right] \\
& +\mathbf{P}\left[\vec{T}(\gamma) \leq C_{8} r, \gamma \text { with less than } C|\gamma| \text { closed edges }\right] \\
\leq \quad & \mathbf{P}\left[\vec{T}(\gamma) \leq C_{8} r, \gamma \text { with more than } C r \text { closed edges }\right]+C_{6} \exp \left(-C_{7} r\right) .
\end{aligned}
$$

For each closed edge $e$, we know that $t(e)>0$. For $\epsilon>0$, we take $\delta>0$ small such that

$$
\mathbf{P}[0<t(e) \leq \delta]=F(\delta)-F(0) \leq \epsilon .
$$

For each closed edge, if it satisfies $t(e) \leq \delta$, we say it is a bad edge. Thus,

$$
\mathbf{P}[e \text { is closed and bad }]=\mathbf{P}[0<t(e) \leq \delta] \leq \epsilon .
$$

Now, on $\{\exists$ an optimal $\gamma$ from $\mathbf{0}$ to $(r, \theta)$ with more than $C|\gamma|$ closed edges $\}$, we estimate the event that there are at least $C r / 2$ bad edges in $\gamma$. By (4.7),

$$
|\gamma| \leq 2 r
$$

Now we fix the path $\gamma$. Since each vertex in $\gamma$ can be adjacent only from a north or an east edge, there are at most $2^{2 r}$ choices for $\gamma$. If $\gamma$ is fixed, there are at most

$$
\sum_{l=1}^{2 r}\left(\begin{array}{c}
2 r \\
l
\end{array}\right) \leq 2^{2 r}
$$

choices for these closed edges. If these closed edges are fixed, as we mentioned above, each edge has a probability less than $\epsilon$ to be also bad. In addition, we also have another $2^{2 r}$ choices to select these bad edges from these closed edges. Therefore, if we take $\epsilon=\epsilon(F, \delta, C)$ small, then there exist positive constants $C_{i}=C_{i}(F, \delta, \theta, C)$ for $i=9,10$ such that

$$
\begin{aligned}
& \mathbf{P}[\exists \text { a NE } \gamma \text { from } \mathbf{0} \text { to }(r, \theta) \text { with more than } C r \text { closed edges, } \\
& \text { these closed edges contain more than } \mathrm{Cr} / 2 \text { bad edges] } \\
& \leq \sum_{l=C r / 2}^{\infty} 2^{2 r} 2^{2 r} 2^{2 r}(\epsilon)^{l} \\
& \leq C_{9} \exp \left(-C_{10} r\right) \text {. }
\end{aligned}
$$

If

$$
\vec{T}(\mathbf{0},(r, \theta)) \leq C_{8} r
$$


for $\theta<\theta_{p}^{-}$, then there is a NE path $\gamma$ from 0 to $(r, \theta)$ with a passage time less than $C_{8} r$. Therefore, by (4.13) and (4.14)

$$
\begin{aligned}
& \mathbf{P}\left[\vec{T}(\mathbf{0},(r, \theta)) \leq C_{8} r\right] \\
\leq \quad & \mathbf{P}[\exists \text { a NE } \gamma \text { from } \mathbf{0} \text { to }(r, \theta) \text { with more than } C r \text { closed edges, } \\
& \text { these closed edges contain less than } \left.C r / 2 \text { bad edges, } \vec{T}(\gamma) \leq C_{8} r\right]+C_{9} \exp \left(-C_{10} r\right) \\
\leq \quad & \mathbf{P}\left[\exists \text { a NE } \gamma \text { from } \mathbf{0} \text { to }(r, \theta), \gamma \text { contains less than } C r / 2 \text { bad edges, } \vec{T}(\gamma) \leq C_{8} r\right] \\
& +C_{9} \exp \left(-C_{10} r\right) .
\end{aligned}
$$

If there is a NE path from 0 to $(r, \theta)$ with less than $C r / 2$ bad edges among these $C r$ closed edges, note that each good edge costs at least passage time $\delta$, so the passage time of the path is more than $\delta \mathrm{Cr} / 2$. Thus, if we select $C_{8}$ such that

$$
C_{8}<C \delta / 2 \text {, }
$$

$\mathbf{P}\left[\exists\right.$ a NE $\gamma$ from 0 to $(r, \theta), \gamma$ contains less than $C r / 2$ bad edges, $\left.\vec{T}(\gamma) \leq C_{8} r\right]=0$.

By (4.15) and (4.16), for $F(0) \geq \vec{p}_{c}, \theta<\theta_{p}^{-}$,

$$
\mathbf{P}\left[\vec{T}(\mathbf{0},(r, \theta)) \leq C_{8} r\right] \leq C_{9} \exp \left(-C_{10} r\right) .
$$

When $\theta>\theta_{p}^{+}$, by symmetry, we still have (4.17). Therefore, Theorems 2 and 4 follow.

\section{$5 \quad$ Inside the percolation cone.}

In Section 5, we assume that $F(0)=p>\vec{p}_{c}$ and $\theta \in\left[\theta_{p}^{-}, \theta_{p}^{+}\right]$. Edge $e$ is called an open or a closed edge if $t(e)=0$ or $t(e)>0$, respectively. We define $\tau(e)=0$ if $t(e)=0$, or $\tau(e)=1$ if $t(e)>0$. We also denote by $\vec{T}_{\tau}(u, v)$ the passage time corresponding to $\tau(e)$. Let

$$
B_{\tau}(t)=\left\{v \in \mathbf{Z}^{2}: \vec{T}_{\tau}(\mathbf{0}, v) \leq t\right\} .
$$

We also assume that $(r \cos \theta, r \sin \theta) \in \mathbf{Z}^{2}$ for $r>0$ and $\theta \in\left[\theta_{p}^{-}, \theta_{p}^{+}\right]$without loss of generality. If $(r, \theta) \in B_{\tau}(t)$, then

$$
\vec{T}_{\tau}(\mathbf{0},(r, \theta)) \leq t
$$

Note that $B_{\tau}(t)$ will eventually cover all the vertices in $\mathbf{R}^{+} \times\left[\theta_{p}^{-}, \theta_{p}^{+}\right]$as $t \rightarrow \infty$, so for any $(r, \theta) \in$ $\mathbf{R}^{+} \times\left[\theta_{p}^{-}, \theta_{p}^{+}\right]$, there exists a $t$ such that $(r, \theta) \in B_{\tau}(t)$. Let $\sigma$ be the smallest $t$ such that $(r, \theta) \in$ $B_{\tau}(t)$. We will estimate $\sigma$ to show that there exist positive constants $C_{i}=C_{i}(F)$ for $i=1$, 2 such that for all large $k$,

$$
\mathbf{P}[\sigma \geq k] \leq C_{1} \exp \left(-C_{2} k\right) \text { uniformly in } r>0 \text { and } \theta \in\left[\theta_{p}^{-}, \theta_{p}^{+}\right] .
$$

Note that

$$
\mathbf{P}[\sigma \geq k]=\sum_{\Gamma} \mathbf{P}\left[\sigma \geq k, B_{\tau}(k-2)=\Gamma\right]
$$

where $\Gamma$, containing the origin, takes all possible vertex sets in the first quadrant. We also remark that for distinct $\Gamma_{1}$ and $\Gamma_{2}$,

$$
\left\{\sigma \geq k, B_{\tau}(k-2)=\Gamma_{1}\right\} \text { and }\left\{\sigma \geq k, B_{\tau}(k-2)=\Gamma_{2}\right\} \text { are disjoint. }
$$


If $\sigma \geq k$ and $B_{\tau}(k-2)=\Gamma$, then $\Gamma$ does not contain $(r, \theta)$ :

$$
\Gamma \cap(r, \theta)=\emptyset \text {. }
$$

In other words, the above sum is over all $\Gamma$ that do not contain $(r, \theta)$. Thus, by Lemma 5 , there is no $\mathrm{NE}$ open path from $\partial_{o}(\Gamma)$ to $(r, \theta)$. Otherwise, $\sigma<k$, which is contrary to the assumption that $\sigma \geq k$. For a fixed $\Gamma$, we denote by $\mathscr{E}_{k}(\Gamma)$ the above event that there is no NE open path from $\partial_{o}(\Gamma)$ to $(r, \theta)$. Thus, there exists a NE open path outside $\Gamma \cup \partial_{e} \Gamma$ from $\partial_{o} \Gamma$ to $(r, \theta)$. Note that $\mathscr{E}_{k}(\Gamma)$ only depends on configurations of edges outside $\Gamma \cup \partial_{e} \Gamma$, so by Lemma 6 , for any fixed $\Gamma$ with $\Gamma \cap(r, \theta)=\emptyset$,

$$
\mathscr{E}_{k}(\Gamma) \text { and }\left\{B_{\tau}(k-2)=\Gamma\right\} \text { are independent. }
$$

By (1.5), there exists $0<\delta<1$, uniformly in $r>0$ and $\theta \in\left[\theta_{p}^{-}, \theta_{p}^{+}\right]$such that for any fixed $\Gamma$ that does not contain $(r, \theta)$,

$$
\mathbf{P}\left[\mathscr{E}_{k}(\Gamma)\right] \leq 1-\mathbf{P}[\mathbf{0} \rightarrow(r, \theta)] \leq 1-\delta
$$

With these observations,

$$
\begin{aligned}
\mathbf{P}[\sigma \geq k] & =\sum_{\Gamma} \mathbf{P}\left[\sigma \geq k, B_{\tau}(k-2)=\Gamma\right] \\
& \leq \sum_{\Gamma} \mathbf{P}\left[B_{\tau}(k-2)=\Gamma, \mathscr{E}_{k}(\Gamma)\right] \\
& \leq \sum_{\Gamma} \mathbf{P}\left[B_{\tau}(k-2)=\Gamma\right](1-\delta),
\end{aligned}
$$

where the sum is over all $\Gamma$ containing the origin but not $(r, \theta)$. Note that for a fixed $\Gamma$ containing the origin but not $(r, \theta)$, by Lemma 5 again,

$$
\left\{B_{\tau}(k-2)=\Gamma\right\} \subset\{\sigma \geq(k-2)\} .
$$

Therefore,

$$
\mathbf{P}[\sigma \geq k] \leq(1-\delta) \mathbf{P}[\sigma \geq(k-2)]
$$

Thus, (5.3) follows if we iterate (5.7). We show Theorem 1 by (5.3). In fact, if $t(e)$ is bounded from above by a constant, then Theorem 1 is implied by (5.3) directly. However, if we restrict ourselves only on a moment condition, the proof is more intricate:

Proof of Theorem 1. Given $\sigma$, the passage time $T(\mathbf{0},(r, \theta))$ is dominated by the sum of $\sigma$ copies of the weight distribution, conditioned to be non-zero. This is because, conditioned on the set of closed edges, the weights of the closed edges are i.i.d. and each has the original weight distribution conditioned on being non-zero. We simply choose an arbitrary path from the origin to $(r, \theta)$ containing $\sigma$ closed edges (for example, choose the first one in the lexicographic order), and sum the weights of the closed edges along that path to get an upper bound on the passage time. Let $Y_{1}, Y_{2}, \cdots$ represent i.i.d. copies of the edge-weight distribution conditioned to be non-zero. If the original weight-distribution has a finite $m$ th moment, then so does this distribution. Since $\sigma$ is only affected by zero-edges, $\left\{Y_{i}\right\}$ and $\sigma$ are also independent. Thus, by (5.3), we get

$$
\begin{aligned}
\mathbf{E} \vec{T}(\mathbf{0},(r, \theta))^{m} & =\mathbf{E}\left[\mathbf{E} \vec{T}(\mathbf{0},(r, \theta))^{m} \mid \sigma\right] \\
& \leq \mathbf{E}\left[\mathbf{E}\left(Y_{1}+Y_{2}+\cdots+Y_{\sigma}\right)^{m}\right] \\
& \leq \mathbf{E}\left[\sigma^{m} \mathbf{E} Y_{1}^{m}\right] \\
& =\mathbf{E} \sigma^{m} \mathbf{E} Y_{1}^{m}<\infty .
\end{aligned}
$$


So Theorem 1 follows.

\section{Critical phase.}

Proof of Theorem 5. In Section 6, we assume that $F(0)=\vec{p}$. There are two possible behaviors for $F$ at 0 :

(a) either there exists a small $h$ such that $F(x)=F(0)=\vec{p}_{c}$ for $x \in[0, h]$,

(b) or there exists a sequence $\left\{x_{n}\right\}$ with $x_{n} \downarrow 0$ such that $F\left(x_{n}\right) \downarrow F(0)$ and $F\left(x_{n}\right)>F(0)$.

Let us assume that case (b) holds. For each $n$, we construct another distribution:

$$
G_{n}(x)= \begin{cases}F(0) & \text { if } 0 \leq x<x_{n} \\ F(x) & \text { if } x_{n} \leq x\end{cases}
$$

By this definition, for each $n$,

$$
G_{n}\left(x_{n}\right)>\vec{p}_{c} .
$$

By (1.5), for all $r$, there exists a directed path from the origin to $(r, \pi / 4)$ such that its passage time in each edge is at most $x_{n}$ with a positive probability. By (1.1), for each $n$,

$$
\vec{\mu}_{G_{n}}(\pi / 4) \leq 2 x_{n} .
$$

By Lemma 7,

$$
\vec{\mu}_{F}(\pi / 4) \leq \vec{\mu}_{G_{n}}(\pi / 4) \leq 2 x_{n}
$$

By (6.2), we can show that

$$
\vec{\mu}_{F}(\pi / 4)=0 .
$$

Therefore, Theorem 5 follows if case (b) holds.

Now we focus on case (a). Note that $F$ cannot be flat forever, so there are points $h_{1}>h>0$ such that $F\left(h_{1}\right)>F(0)$ and $F(x)=F(0)$ for $0 \leq x \leq h$. Now we assume that $F$ satisfies the following extra condition:

(i). There exists $h>0$ such that

$$
F(x)=F(0)=\vec{p}_{c} \text {, when } 0 \leq x<h \text {, and } F(x)>\vec{p}_{c} \text {, when } x \geq h .
$$

In other words, there is a jump point at $h$.

We focus on case (a) (i). We take $\epsilon>0$ small such that

$$
F(0)+\epsilon<F(h)
$$

Then we construct another distribution:

$$
G_{\epsilon}(x)=\left\{\begin{array}{cc}
F(0)+\epsilon & \text { if } 0 \leq x<h, \\
F(x) & \text { if } h \geq x .
\end{array}\right.
$$

As we defined, $t(e)$ is the random variable with distribution $F$. Let $g_{\epsilon}(e)$ be the random variable with distribution $G_{\epsilon}$. We couple $t(e)$ and $g_{\epsilon}(e)$ and then assume that all the couples $\left(t(e), g_{\epsilon}(e)\right)_{e \in \mathbf{L}^{2}}$ are independent and identically distributed. Define $g_{\epsilon}(e)$ as follows: 
If $t(e)=0$, then $g_{\epsilon}(e)=0$.

If $t(e)=x>h$, then $g_{\epsilon}(e)=x$.

If $t(e)=h$, then

$$
g_{\epsilon}(e)\left\{\begin{array}{l}
=0 \\
=h \quad \text { with probability } \epsilon\left(F(h)-\vec{p}_{c}\right)^{-1}, \\
\text { with probability } 1-\epsilon\left(F(h)-\vec{p}_{c}\right)^{-1} .
\end{array}\right.
$$

We need to verify that $g_{\epsilon}$ has distribution $G_{\epsilon}$. Since the verification is simple, we leave to the readers.

Now we show Theorem 5 under case (a) (i). Let $\gamma_{t}$ be an optimal path for $\vec{T}_{t}(\mathbf{0},(r, \pi / 4))$ with time state $t(e)$, and let $\gamma_{g_{\epsilon}}$ be an optimal path for $T_{g_{\epsilon}}(0,(r, \pi / 4))$ with time state $g_{\epsilon}(e)$. Here, for each configuration, we select $\gamma_{g_{\epsilon}}$ in a unique method. For each edge $e \in \gamma_{g_{\epsilon}}$, we consider passage time $t(e)$. If $t(e)>h$, then $g_{\epsilon}(e)=t(e)$ by our definition. In addition, if $t(e)=h$, it also follows from the definition that $g_{\epsilon}(e)=h$ or $g_{\epsilon}(e)=0$. Therefore,

$$
\vec{T}_{t}(\mathbf{0},(r, \pi / 4)) \leq \vec{T}\left(\gamma_{g_{\epsilon}}\right)+h \sum_{e \in \gamma_{g_{\epsilon}}} I_{\left(t(e)=h, g_{\epsilon}(e)=0\right)} .
$$

By (6.4),

$$
\mathbf{E} \vec{T}_{t}(\mathbf{0},(r, \pi / 4)) \leq \mathbf{E} \vec{T}\left(\gamma_{g_{\epsilon}}\right)+h \sum_{\beta} \sum_{e \in \beta} \mathbf{P}\left[t(e)=h, g_{\epsilon}(e)=0, \gamma_{g_{\epsilon}}=\beta\right]
$$

where the first sum in (6.5) is over all possible NE paths $\beta$ from 0 to $(r, \pi / 4)$. Let us estimate

$$
\sum_{\beta} \sum_{e \in \beta} \mathbf{P}\left[t(e)=h, g_{\epsilon}(e)=0, \gamma_{g_{\epsilon}}=\beta\right]
$$

Note that the value of $t(e)$ may depend on the value of $g_{\epsilon}(e)$, but not on the other values of $g_{\epsilon}(b)$ for $b \neq e$, so by our definition,

$$
\begin{aligned}
& \mathbf{P}\left[t(e)=h, g_{\epsilon}(e)=0, \gamma_{g_{\epsilon}}=\beta\right]=\mathbf{P}\left[t(e)=h \mid g_{\epsilon}(e)=0, \gamma_{g_{\epsilon}}=\beta\right] \mathbf{P}\left[g_{\epsilon}(e)=0, \gamma_{g_{\epsilon}}=\beta\right] \\
\leq & \mathbf{P}\left[t(e)=h \mid g_{\epsilon}(e)=0\right] \mathbf{P}\left[\gamma_{g_{\epsilon}}=\beta\right]=\epsilon\left[\vec{p}_{c}\left(F(h)-\vec{p}_{c}\right)\right]^{-1} \mathbf{P}\left[\gamma_{g_{\epsilon}}=\beta\right] .
\end{aligned}
$$

Note that $\beta$ has at most $2 r$ edges, so by using (6.6) there exists $C$ such that

$$
\sum_{\beta} \sum_{e \in \beta} \mathbf{P}\left[t(e)=h, g_{\epsilon}(e)=0, \gamma_{g_{\epsilon}}=\beta\right] \leq \sum_{\beta} \sum_{e \in \beta} C \epsilon \mathbf{P}\left[\gamma_{g_{\epsilon}}=\beta\right] \leq 2 C \epsilon r .
$$

By (6.5) and (6.7), there exists $C=C(F)$ such that

$$
\mathbf{E} \frac{\mathbf{T}_{t}(\mathbf{0},(r, \pi / 4))}{r} \leq \mathbf{E} \frac{\vec{T}_{g_{\epsilon}}(\mathbf{0},(r, \pi / 4))}{r}+C \epsilon
$$

We take $r \rightarrow \infty$ in (6.8) to have

$$
\vec{\mu}_{F}(\pi / 4) \leq \vec{\mu}_{G_{\epsilon}}(\pi / 4)+C \epsilon .
$$

Note that $G_{\epsilon}(0)>\vec{p}_{c}$, so by Corollary 3 and (6.9),

$$
\vec{\mu}_{F}(\pi / 4)=0 .
$$


Therefore, Theorem 5 follows under case (a) (i).

Finally, we focus on case (a) without other assumptions. As we mentioned, $t(e)$ is not a constant. Thus, there exists $h_{1}>h$ such that $F\left(h_{1}\right)>F(0)$. We construct

$$
H(x)=\left\{\begin{array}{cc}
F(0) & \text { if } 0 \leq x<h_{1} \\
F(x) & \text { if } h_{1} \leq x
\end{array}\right.
$$

With this definition,

$$
H \leq F \text {, and } H(0)=\vec{p}_{c},
$$

and $H(x)$ has a jump point at $h_{1}$. By the analysis of case (a) (i), we have

$$
\vec{\mu}_{H}(\pi / 4)=0 \text {. }
$$

By Lemma 7,

$$
\vec{\mu}_{F}(\pi / 4) \leq \vec{\mu}_{H}(\pi / 4)=0 .
$$

Thus, Theorem 5 under case (a) follows from (6.11). If we put cases (a) and (b) together, Theorem 5 follows.

Proof of Theorem 7. In this proof, we assume that $t(e)$ only takes 0 (open) and 1 (closed) with probability $\vec{p}_{c}$ and $1-\vec{p}_{c}$, respectively. Let $L_{r}$ be the line $y=-x+r$ inside the first quadrant. Note that $L_{0}$ is just the origin. Bezuidenhout and Grimmett (1990) showed that there is no infinite NE zero-path on $\mathbf{Z}^{2}$. Thus, for fixed $L_{r_{1}}$

$$
\mathbf{P}\left[L_{r_{1}} \rightarrow \infty\right]=0 .
$$

By (6.18), there exists $0<\delta<1$ and $r_{2}=r_{2}\left(r_{1}\right)$ such that

$$
\mathbf{P}\left[L_{r_{1}} \nrightarrow L_{r_{2}}\right] \geq \delta .
$$

If $L_{r_{1}} \nrightarrow L_{r_{2}}$, then NE path from $L_{r_{1}}$ to $L_{r_{2}}$ has to use at least one edge with passage time 1 . Let $I\left(L_{r_{1}}, L_{r_{2}}\right)$ be the indicator of the event that there is no NE open path from $L_{r_{1}}$ to $L_{r_{2}}$. For large $r$, let $r_{1} \leq r_{2} \leq \cdots \leq r_{m} \leq r$ be a sequence such that for $i<m-1$,

$$
\mathbf{P}\left[L_{r_{i}} \not \rightarrow L_{r_{i+1}}\right] \geq \delta .
$$

Note that any NE path from the origin to $(r, \pi / 4)$ has to cross the strip between $L_{i}$ and $L_{i+1}$ for $i=1, \cdots, m-1$, so

$$
\mathbf{E} \vec{T}(\mathbf{0},(r, \pi / 4)) \geq \mathbf{E} \sum_{i=1}^{m} I\left(L_{r_{1}}, L_{r_{2}}\right)=\delta m .
$$

By (6.19), we have $m \rightarrow \infty$ as $r \rightarrow \infty$. Therefore, by (6.21),

$$
\lim _{r \rightarrow \infty} \mathbf{E} \vec{T}(\mathbf{0},(r, \pi / 4))=\infty .
$$

Theorem 7 follows from (6.22).

Acknowledgments. The author acknowledges the detailed valuable comments from referees which simplified proofs and resulted in an improved exposition. 


\section{References}

[1] Alexander, K. (1996). Approximation of subadditive functions and convergence rates in limiting-shape results. Ann. Probab. 25, 30-55.

[2] Bezuidenhout, C. and Grimmett, G. (1990). The critical contact process dies out. Ann. Probab. 18, 1462-1482.

[3] Chayes, J., Chayes, L. and Durrett, R. (1986). Critical behavior of the two dimensional first passage time, J. Stat. Phys. 45, 933-948.

[4] Cox, T. and Kesten, H. (1981). On the continuity of the time constant of first-passage percolation, J. Appl. Probab. 18, 809-819.

[5] Durrett, R. (1984). Oriented percolation in two dimensions. Ann. Probab. 12, 999-1040.

[6] Durrett, R. and Liggett, T. (1981). The shape of the limit set in Richardson's growth model. Ann. Probab. 9, 186-193.

[7] Grimmett, G. (1999). Percolation. Springer, Berlin.

[8] Hammersley, J. M. and Welsh, D. J. A. (1965). First-passage percolation, subadditive processes, stochastic networks and generalized renewal theory. In Bernoulli, Bayes, Laplace Anniversary Volume (J. Neyman and L. LeCam, eds.) 61-110. Springer, Berlin.

[9] Kesten, H. (1986). Aspects of first-passage percolation. Lecture Notes in Math. 1180, 125-264. Springer, Berlin.

[10] Kesten, H. and Zhang, Y. (1990). The probability of a large finite cluster in supercritical Bernoulli percolation. Ann. Probab. 18, 537-555.

[11] Kesten, H. and Zhang, Y. (1997). A central limit theorem for critical first passage percolation in two dimensions. Probab. Theory Related Fields. 107 137-160.

[12] Marchand, R. (2002). Strict inequalities for the time constant in first passage percolation. Ann. Appl. Probab. 12, 1001-1038.

[13] Martin, J. (2004). Limit shape for directed percolation models. Ann. Probab. 32 2908-2937.

[14] Smythe, R. T. and Wierman, J. C. (1978). First passage percolation on the square lattice. Lecture Notes in Math. 671. Springer, Berlin.

[15] Talagrand, M. (1995). Concentration of measure and isoperimetric inequalities in product spaces. Inst. Hautes Publ. Math. Etudes Sci. Publ. Math. 81, 73-205.

[16] Yukich, J. and Zhang, Y. (2006). Singularity points for first passage percolation. Ann. Probab. 34, 577-592.

[17] Zhang, Y. (1995). Supercritical behaviors in first-passage percolation. Stoch. Proc. Appl. 59, 251-266. 
[18] Zhang, Y. (1999). Double behavior of critical first passage percolation. In Perplexing Problems in Probability (M. Bramson and R. Durrett eds.) 143-158. Birkhauser, Boston.

[19] Zhang, Y. (2006). The divergence of fluctuations for shape in first passage percolation. Probab. Theory Related Fields. 136, 298-320.

[20] Zhang, Y. (2008). Shape fluctuations are different in different directions. Ann. Probab. 36, $331-362$. 